\title{
Aprendizaje y su relación en la motivación al logro de estudiantes universitarios
}

\section{Learning and its relation in the motivation to the achievement of university students}

\author{
Gabriel Eduardo Alvarado-Azaña \\ gabriel.alvarado@unmsm.edu.pe \\ Universidad Nacional Mayor de San Marcos, Lima \\ Perú \\ https://orcid.org/0000-0003-0883-3472
}

Recepción: 24 septiembre 2020

Revisado: 25 de octubre 2020

Aprobación: 16 noviembre 2020

Publicación: 07 de diciembre 2020 


\begin{abstract}
RESUMEN
La investigación centró su objetivo en analizar el aprendizaje y su relación en la motivación al logro de estudiantes universitarios del Perú. Se generó en consideración al método analítico - sintético y un tipo de descriptivo documental con diseño bibliográfico. La motivación al logro potenciada desde los valores étnicos de los pueblos ancestrales, contribuye a fomentar mayor resiliencia en el estudiante, a luchar no solo por adquirir un conocimiento que le permita aprobar un determinado periodo académico, sino, concebir la educación como un proceso de continua transformación para su ser y realidad social circundante. El docente debe trabajar fundamental la motivación al logro como un factor generador de autonomía al aprendizaje en el estudiante.
\end{abstract}

Descriptores: Autoaprendizaje; aprendizaje activo; voluntad de realización. (Palabras tomadas del Tesauro UNESCO).

\begin{abstract}
The research focused its objective on analyzing learning and its relationship in motivation to achieve university students in Peru. It was generated in consideration of the analyticalsynthetic method and a type of documentary descriptive with bibliographic design. The motivation to achieve enhanced from the ethnic values of the ancestral peoples, contributes to promoting greater resilience in the student, to fight not only to acquire knowledge that allows them to pass a certain academic period, but to conceive education as a process of continuous transformation for your being and surrounding social reality. The teacher must work fundamentally on motivation to achieve as a factor that generates autonomy for student learning.
\end{abstract}

Descriptors: Self instruction; activity learning; achievement motivation. (Words taken from the UNESCO Thesaurus). 


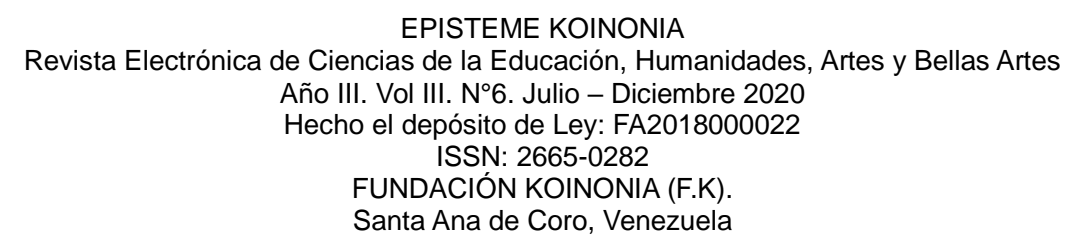

Gabriel Eduardo Alvarado-Azaña

\section{INTRODUCCIÓN}

El aprendizaje es un proceso ligado a diversas operaciones mentales, conductuales, sociales, en esencia, se procura que la persona interprete una realidad o hecho, aprenda y pueda actuar repetidamente a partir de esa experiencia, por lo que se encuentra ligado a lo cognitivo - conductual del ser humano (Pérez-Ariza \& Hernández-Sánchez, 2014). De ese modo, se motiva a aprender con la finalidad de modificar patrones o simplemente incorporar nuevas habilidades, competencias, para constituirse en mejor persona, estudiante, profesional.

Por consiguiente, el aprendizaje proyecta la generación de un estudiante dinámico y en evolución a lo largo de un determinado período de tiempo, es así, que, por ejemplo, al inicio de un período académico, los estudiantes ingresan con ciertos conocimientos, al final de ese periodo, se espera que tenga mayor - mejor conocimiento sobre lo abordado, no solo desde el punto de vista cognitivo, sino, experiencial, de ese modo, podrá comprobarse la existencia de modificación en el estudiante, por consiguiente lo aprendido, modificado, debe considerarse como aprendizaje.

Es así que el aprendizaje es concebido por diversas corrientes de la psicología educativa en función de la esencia filosófica de la misma, de allí se genera una cosmovisión de abordar y evaluar los aprendizajes, a través de una planificación tecno curricular enfocada a propiciar una didáctica favorecedora del conocimiento, a través de estrategias de aprendizaje diseñadas con la finalidad de promover un mejor rendimiento académico (García-Gajardo, et al., 2015).

En este sentido, el componente psicológico juega un rol importante en la educación, por cuanto el docente al abordar este aspecto del estudiante, puede motivarlo al logro de un mejor aprendizaje, por ende, rendimiento académico; es importante conocer que la postura de (Morán-Astorga \& Menezes-dos-Anjos, 2016), concibe a los estudiantes universitarios con motivación al logro, como de mayor seguridad y serenidad a la hora de afrontar los retos académicos, esto conlleva a pensar en la posibilidad de que realmente se requiere profundizar por parte de los docentes, la motivación al logro en los estudiantes 


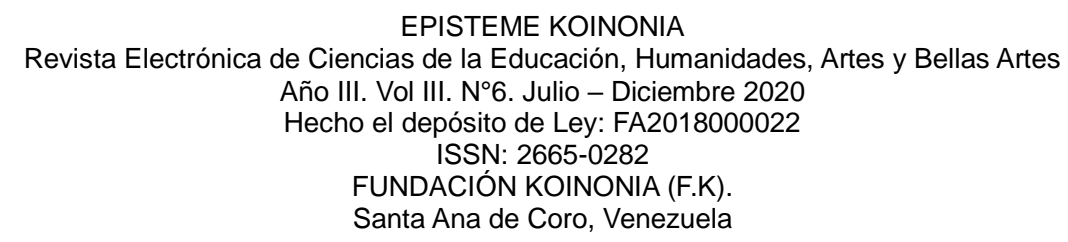

Gabriel Eduardo Alvarado-Azaña

universitarios, por cuanto se corresponde a una edad biológica donde debería existir la madurez necesaria para asumir el compromiso de formarse responsablemente, incluso de ser auto didácticas de su propio aprendizaje.

Siendo la tendencia actual en la educación global, es decir, la del aprendizaje autónomo, además de la incorporación de las tecnologías de la información y comunicación (TIC), no solo como un recurso, sino, como una opción pedagógica para desarrollar la educación de cara a la era digital y de inteligencia artificial, se hace aun mayor la necesidad de incentivar la motivación al logro, dado que los nuevos enfoques curriculares basados en el constructivismo social, son esencialmente centrados en el estudiante y no en el docente (Loaiza-Villalba, et al., 2019).

Es por esta razón, que la actual investigación centra su objetivo en analizar el aprendizaje y su relación en la motivación al logro de estudiantes universitarios del Perú.

\section{MÉTODO}

La investigación se generó en consideración al método analítico - sintético y un tipo de descriptivo documental con diseño bibliográfico, tomándose una muestra de artículos de investigación publicados en revistas arbitradas con referencia a las variables de estudio, de ese modo, se procedió en interpretar las partes para consolidar una síntesis teórica como propuesta para trabajar la motivación al logro desde diversas aristas socio culturales didácticas, siendo la técnica de análisis de contenido, la empleada para tal fin.

\section{RESULTADOS}

En razón de las investigaciones escrutadas se presenta:

La postura de (Alemán-Marichal, et al., 2018), gira en torno al rol docente de conocer la motivación de sus estudiantes, con la finalidad de construir estrategias didácticas, en razón de contribuir no solo a un mejor aprendizaje, sino, a un crecimiento personal favorable en la consecución de metas individuales y educativas, de ese modo, el docente contribuye con promover no solo un excelente estudiante, sino, ciudadano. 


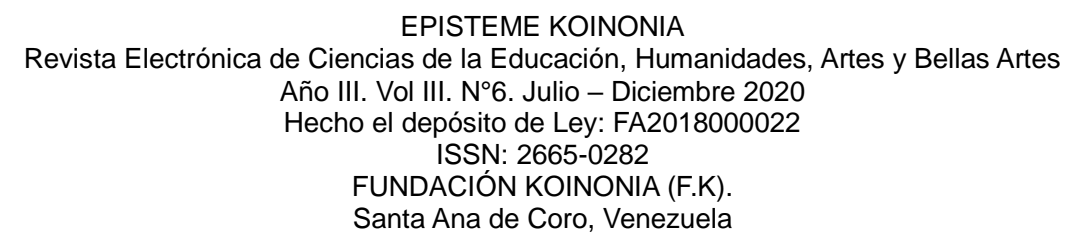

Gabriel Eduardo Alvarado-Azaña

Prosiguiendo con esta concepción, (Tirado, et al., 2013), presentan en sus conclusiones que los estudiantes que tienen una cultura personal arraigada al logro, presentan mejor rendimiento académico por encima de aquellos que tienen una condición socio económica de mayor estabilidad, denotándose en este aspecto que cuando el estudiante se siente motivado internamente a conseguir metas académicas, es capaz de lograrlas a pesar de sus limitaciones económicas, situación que conlleva a generar en la relación docente - estudiantes, dedicación a fomentar la motivación al logro.

En referencia a lo descrito, se hace necesario contar con docentes con apertura a incorporar en sus clases, aspectos formativos relacionados al crecimiento personal, esto implica dedicar un poco de tiempo para generar sensibilización sobre la importancia de construir un aprendizaje a partir de una motivación configurada desde la personalidad del estudiante, requiriéndose un currículo flexible - dinámico para que esta práctica pedagógica no sea aislada, sino, forme parte formal en el sistema educativo.

La flexibilidad curricular en la educación, no es una propuesta novedosa por cuanto data del siglo pasado como una propuesta emergente ante el currículo clásico (Acuña-Castillo, 2019)., sin embargo, la misma no ha sido adoptada en la cotidianidad educativa, por cuanto se percibe aun en práctica el modelo estático enciclopedista del modelo tradicional, este enfoque precisamente es que trunca la existencia de trabajar el crecimiento personal como un todo y no de forma atomizada a partir de la iniciativa de algunos docentes.

Por lo tanto, el tema que se plantea sobre aprendizaje y motivación, encierra diversas aristas para desentrañar el fondo de la educación, la cual se basa primordialmente en seguir reproduciendo el modelo cognitivo, a pesar de las propuestas pedagógicas centradas en el estudiante como centro del proceso y no el docente (Burgos-Briones, et al., 2019), es así que (Gargallo-López, et al., 2017), comentan que este enfoque está empleándose con mayor frecuencia en la educación superior, considerada efectiva por parte de los estudiantes a partir del aprendizaje cooperativo - colaborativo que realizan. 
EPISTEME KOINONIA

Revista Electrónica de Ciencias de la Educación, Humanidades, Artes y Bellas Artes

Año III. Vol III. N6. Julio - Diciembre 2020

Hecho el depósito de Ley: FA2018000022

ISSN: 2665-0282

FUNDACIÓN KOINONIA (F.K).

Santa Ana de Coro, Venezuela

Gabriel Eduardo Alvarado-Azaña

Lo cual devela la diferencia entre el enfoque centrado en el docente y en centrado en el estudiante, a continuación, en la figura 1 y 2 , se evidencian:

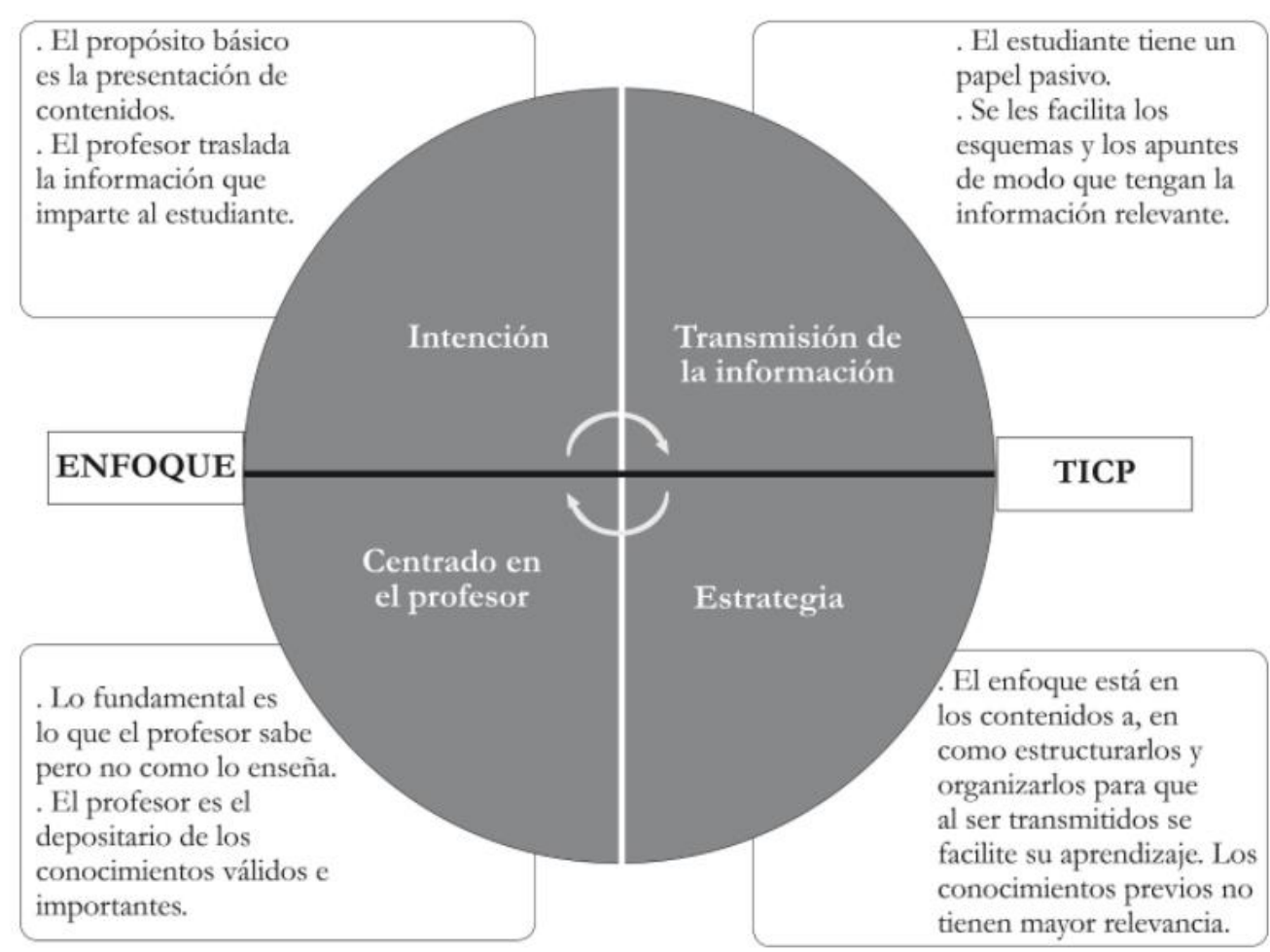

Figura 1: Enfoque centrado en el docente.

Fuente: Soler, et al. (2018).

Básicamente en este enfoque, el profesor se constituye en depositario de la verdad en el estudiante, por consiguiente, la motivación girará en que él estudiante aprenda y reproduzca lo más semejante posible los contenidos o temas abordados por el docente, de ese modo, se garantiza un eficaz proceso de aprendizaje, por ende, un alto rendimiento académico, en resumen, este es un modelo meramente de enseñanza. 
Gabriel Eduardo Alvarado-Azaña

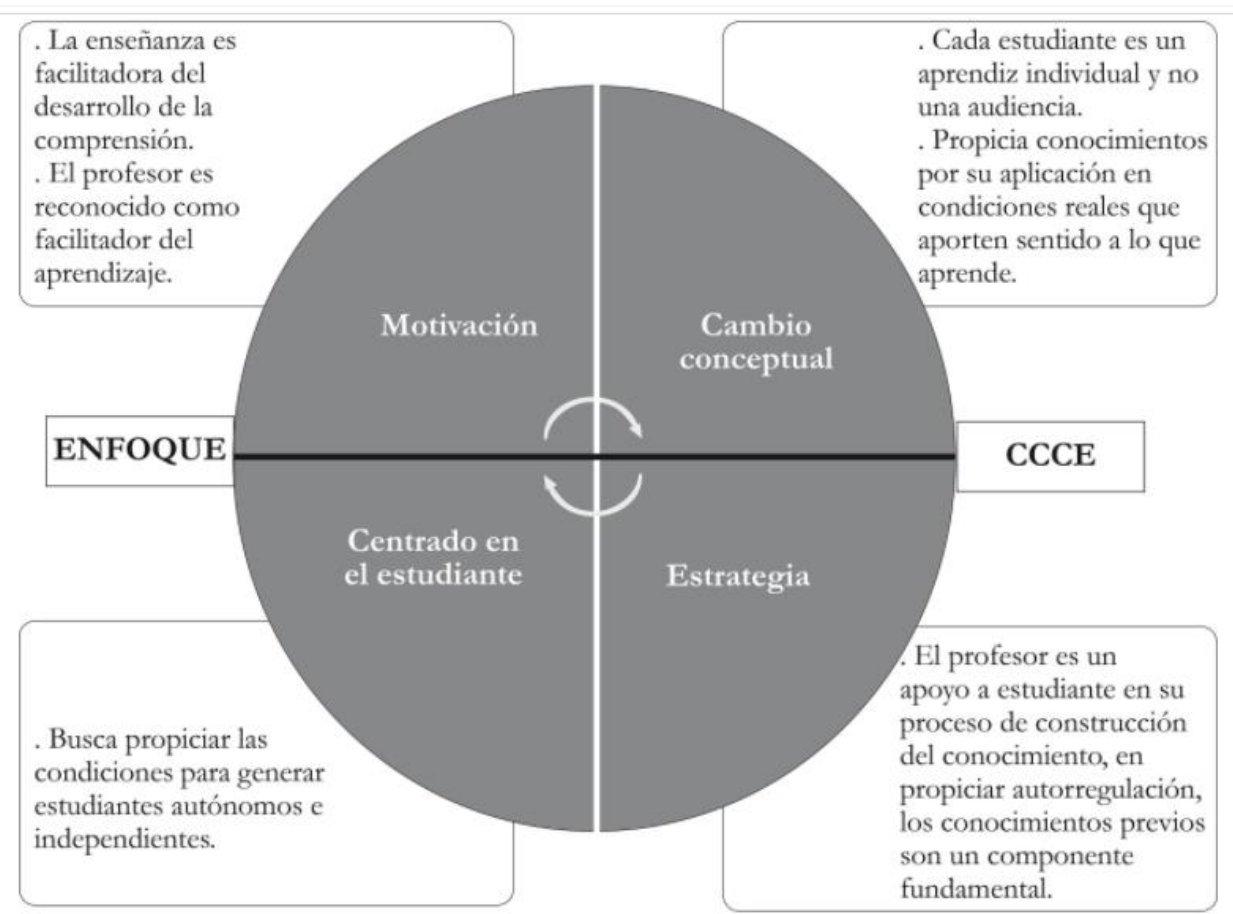

Figura 2: Enfoque centrado en el estudiante.

Fuente: Soler, et al. (2018).

En este enfoque, el docente debe trabajar fundamental la motivación al logro como un factor generador de autonomía al aprendizaje en el estudiante, es un modelo donde la mayor responsabilidad recae en el estudiante, el docente es un mediador entre el aprendizaje y estudiante, prácticamente asume la posición de supervisar que el estudiante construya conocimiento cónsono con la realidad educativa -social, así las estrategias didácticas deben girar en promover el auto aprendizaje, o simplemente lo que se comprende como aprender a lo largo de la vida.

El aprender a lo largo de la vida, es concebido por (Beltrán-Llavador, 2015), como una oportunidad para construir una mejor personalidad a partir de una educación más flexible y abierta a los cambios, esto implica educar para una sociedad sostenible, es decir, en optimización de los recursos, incluyendo los naturales como sostenibilidad en razón de configurar una postura epistémica para la conexión del conocimiento ancestral y 


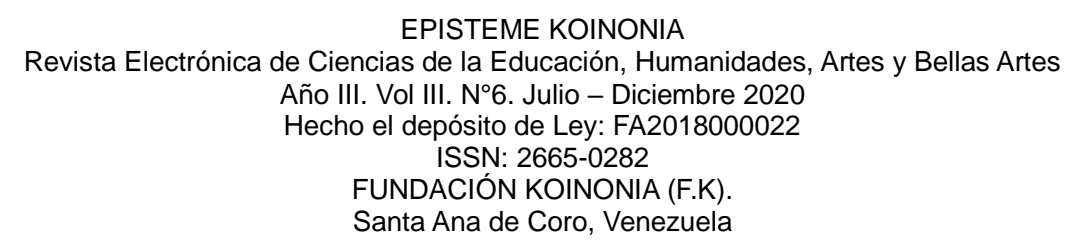

Gabriel Eduardo Alvarado-Azaña

científico, siendo rol del docente, motivar al estudiante a un aprendizaje cultural amplio de su realidad global.

La conjugación de aprender la conexión ancestral - científica (Salgado-Medina, et al., 2018), permite al docente incentivar en el estudiante, su identidad cultural, es empoderar desde su cultura, una motivación al logro para transcender en un mundo que se encauza en borrar las huellas históricas de los pueblos, los cuales han demostrado desde una conducta resiliente, motivarse para conquistar metas comunes (García-Serrano, 2014), ejemplo que debe ser tenido en cuenta en los ambientes de aprendizaje universitario como una enseñanza intercultural para el logro de propósitos colectivos.

Es así que la motivación al logro potenciada desde los valores étnicos de los pueblos ancestrales, contribuye a fomentar mayor resiliencia en el estudiante, a luchar no solo por adquirir un conocimiento que le permita aprobar un determinado periodo académico, sino, concebir la educación como un proceso de continua transformación para su ser y realidad social circundante, ante lo cual, (Villalba-Condori \& Avello-Martínez, 2019), afirman que la resiliencia se relaciona con la satisfacción de la vida.

De ese modo, se tiene que el aprendizaje y la motivación al logro, son variables concomitantes en la aplicación efectiva del enfoque centrado en el estudiante en la educación universitaria, por cuanto no puede existir una asertiva construcción del conocimiento en correspondencia a la premisa constructivista social, la cual sustenta el mencionado enfoque, si el estudiante no se encuentra formalmente motivado a ser el protagonista del proceso.

\section{CONCLUSIÓN}

Las estrategias de mayor eficacia para promover el aprendizaje desde la motivación al logro en estudiantes universitarios, deben focalizarse en generar la confianza para asumir el protagonismo del enfoque centrado en el estudiante, el cual se basa en el constructivismo social y en métodos como aula invertida, entre otros, por consiguiente para ser efectivos en este enfoque, el estudiante requiere ser responsable de la 
construcción de su auto aprendizaje en concordancia con asumir el cumplimiento de metas personales, académicas, profesionales, ciudadanas, en razón de cooperar en un conocimiento intercultural de la realidad social global.

\section{FINANCIAMIENTO}

No monetario

\section{AGRADECIMIENTOS}

A la Universidad Nacional Mayor de San Marcos; por el apoyo en la realización de esta investigación.

\section{REFERENCIAS}

Acuña-Castillo, J. A. (2019). El currículo flexible en la educación artística bogotana: ¿una experiencia de equidad o inequidad? [The flexible curriculum in Bogota's arts education: an experience of equity or inequity?]. Papel Político, 24(1), 1-48. https://doi.org/10.11144/Javeriana.papo24-1.cfea

Alemán-Marichal, B, Navarro-de-Armas, O, Suárez-Díaz, R, Izquierdo-Barceló, Y, \& Encinas-Alemán, T. (2018). La motivación en el contexto del proceso enseñanzaaprendizaje en carreras de las Ciencias Médicas [Motivation in the context of the teaching-learning process in specialties of the Medical Sciences [. Revista Médica Electrónica, 40(4), 1257-1270.

Beltrán-Llavador, J. (2015). Educación a lo largo de la vida: un horizonte de sentido [Education throughout life: a horizon of meaning]. Sinéctica, (45), 01-11.

Burgos-Briones, J., Alvarado-Pino, L., \& Valdez-Guerrero, R. (2019). Enfoque escolar centrado en el estudiante [Student-centered school approach]. CIENCIAMATRIA, 5(1), 45-58. https://doi.org/10.35381/cm.v5i1.240

García-Gajardo, F, \& Fonseca-Grandón, G, \& Concha-Gfell, L. (2015). Aprendizaje y rendimiento académico en educación superior: un estudio comparado [Learning and academic achievement in higher education: a comparative study]. Revista Electrónica "Actualidades Investigativas en Educación", 15(3),1-26. 


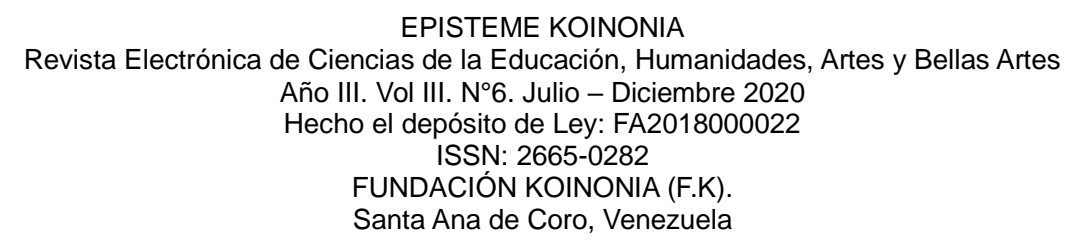

Gabriel Eduardo Alvarado-Azaña

García-Serrano, F. (2014). Territorialidad y autonomía, proyectos minero-energéticos y consulta previa: el caso de los pueblos indígenas de la Amazonia ecuatoriana [Territoriality and autonomy, mining-energy projects and prior consultation: the case of the indigenous peoples of the Ecuadorian Amazon]. Anthropologica, 32(32), 71-85.

Gargallo-López, B, \& Pérez-Pérez, C, \& Jiménez-Rodríguez, M, \& Martínez-Hervás, N, \& Giménez-Beut, J. (2017). Métodos centrados en el aprendizaje, implicación del alumno y percepción del contexto de aprendizaje en estudiantes universitarios [Methods focused on learning, student involvement and perception of the learning context in university students]. Educación XX1, 20(2),161-187.

Loaiza-Villalba, N., García-Botero, J., \& Botero-Restrepo, M. A. (2019). Fortalecimiento del aprendizaje autónomo de estudiantes de licenciatura en lenguas extranjeras colombianos a partir de la teoría de los estilos de aprendizaje, las TIC y el aula invertida [Strengthening the autonomous learning of undergraduate students in Colombian foreign languages from the theory of learning styles, ICT and the flipped classroom]. Revista Boletín 154-169. https://doi.org/10.36260/rbr.v8i9.819

Morán-Astorga, C, \& Menezes-dos-Anjos, E. (2016). La motivación de logro como impulso creador de bienestar: su relación con los cinco grandes factores de la personalidad [The achievement motivation as a creative impulse of well-being: its relation with the five great factors of the personality]. International Journal of Developmental and Educational Psychology, 2(1),31-40.

Pérez-Ariza, K, \& Hernández-Sánchez, J. (2014). Aprendizaje y comprensión. Una mirada desde las humanidades [Learning and comprehension. A glance from the humanities]. Humanidades Médicas, 14(3), 699-709.

Salgado-Medina, R, Keyser-Ohrt, U, \& Ruizde-La-Torre, G. (2018). Conocimientos y saberes locales en tres propuestas curriculares para educación indígena [Local knowledge in three curricular proposals for indigenous education]. Sinéctica, (50). https://doi.org/10.31391/s2007-7033(2018)0050-003

Soler, M, Cárdenas, F, \& Hernández-Pina, F. (2018). Enfoques de enseñanza y enfoques de aprendizaje: perspectivas teóricas promisorias para el desarrollo de investigaciones en educación en ciencias [Teaching and learning approaches: theoretical perspectives to develop research in science education]. Ciência \& Educação $\quad$ (Bauru), 24(4), $\quad$ 993-1012. https://doi.org/10.1590/1516$\underline{731320180040012}$ 
EPISTEME KOINONIA

\author{
Revista Electrónica de Ciencias de la Educación, Humanidades, Artes y Bellas Artes \\ Año III. Vol III. Nº6. Julio - Diciembre 2020 \\ Hecho el depósito de Ley: FA2018000022 \\ ISSN: 2665-0282 \\ FUNDACIÓN KOINONIA (F.K). \\ Santa Ana de Coro, Venezuela \\ Gabriel Eduardo Alvarado-Azaña
}

Tirado, F, Santos, G, \& Tejero-Díez, D. (2013). La motivación como estrategia educativa: Un estudio en la enseñanza de la botánica [Motivation as an educational strategy. A study about teaching Botany]. Perfiles educativos, 35(139), 79-92.

Villalba-Condori, K, \& Avello-Martínez, R. (2019). Resiliencia como factor determinante para la satisfacción con la vida en estudiantes universitarios [Resilience as a factor determining satisfaction with life among university students]. Educación Médica Superior, 33(3), e1845.

(C2020 por el autor. Este artículo es de acceso abierto y distribuido según los términos y condiciones de la licencia Creative Commons Atribución-NoComercial-Compartirlgual 4.0 Internacional (CC BY-NC-SA 4.0) (https://creativecommons.org/licenses/by-nc-sa/4.0/). 